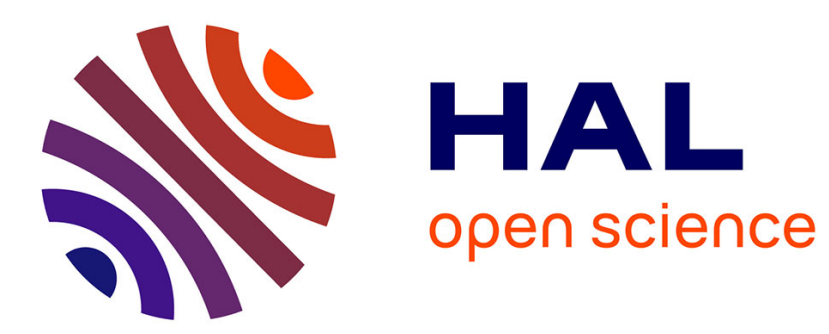

\title{
Real-time numerical dosimetry of low-frequency electromagnetic fields by using multipoles
}

Francois Tavernier, Riccardo Scorretti, Noël Burais, Hubert Razik, Jean-Yves

Gaspard

\section{- To cite this version:}

Francois Tavernier, Riccardo Scorretti, Noël Burais, Hubert Razik, Jean-Yves Gaspard. Real-time numerical dosimetry of low-frequency electromagnetic fields by using multipoles. IEEE Transactions on Magnetics, inPress, 10.1109/TMAG.2021.3065554 . hal-03189650

\section{HAL Id: hal-03189650 \\ https://hal.science/hal-03189650}

Submitted on 9 Apr 2021

HAL is a multi-disciplinary open access archive for the deposit and dissemination of scientific research documents, whether they are published or not. The documents may come from teaching and research institutions in France or abroad, or from public or private research centers.
L'archive ouverte pluridisciplinaire HAL, est destinée au dépôt et à la diffusion de documents scientifiques de niveau recherche, publiés ou non, émanant des établissements d'enseignement et de recherche français ou étrangers, des laboratoires publics ou privés. 


\title{
Real-time numerical dosimetry of low-frequency electromagnetic fields by using multipoles
}

\author{
François Tavernier ${ }^{1}$, Riccardo Scorretti ${ }^{1}$, Noël Burais ${ }^{1}$, Hubert Razik ${ }^{1}$, and Jean-Yves Gaspard ${ }^{2}$ \\ ${ }^{1}$ Univ Lyon, UCBL, ECL, CNRS, AMPERE, F-69622 Villeurbanne, France \\ ${ }^{2}$ Magtech, Gleizé, F-69400, France
}

\begin{abstract}
Computation of the internal electric field induced in the human body by low-frequency power systems is time consuming. We compute an approximate solution in nearly-real time by using a reduced basis for the internal field. The basis is computed once (off-line) by approximating the source by multipoles, the coefficients of which can be computed for any position of the human body thanks to analytic translation and rotation formulas. The results are validated with respect of Finite Element (FEM) computation.
\end{abstract}

Index Terms-Dosimetry, Spherical harmonics, Finite elements.

\section{INTRODUCTION}

Human exposure to electromagnetic fields is a source of concern, and has to be limited in order to prevent health adverse effects. In particular, guidelines have been developed by ICNIRP [1], [2] and by IEEE [3]. Apart from differences in the permitted levels and other details, these guidelines are similar: both of them are two-tiers standards (different limites are defined for workers and general population) and only well known short term effects of exposure to electromagnetic fields have been considered in the definition of the limits. At low frequencies (under $100 \mathrm{kHz}$ ) the only acknowledged direct adverse effect is nerve stimulation. Basic restriction (BR) are given in terms of non measurable internal fields, namely the electric field [1], [3] and the current density [2]. In order to provide more practical limits to the exposure, reference levels (RL) for the magnetic field are defined. The respect of RL guarantees the compliance with $\mathrm{BR}$ as well; in the opposite case, internal fields have to be computed numerically. Hence it is important to quantify the internal electric field induced in the human body by external fields.

At low frequency magnetic exposure, the standard assumptions are that displacement currents are negligible, and the secondary magnetic field generated by induced currents in the human body is negligible as well [4]. By the way, the fact that the reaction field is negligible is one of the reasons for which, so far, it has been impossible to validate experimentally dosimetric computations. In most cases, the equation which is numerically solved in the human body $\Omega$ is:

$$
\operatorname{div}[\sigma(j \omega \mathbf{a}+\operatorname{grad} v)]=0 \quad ; \quad \mathbf{n} \cdot \mathbf{j}=0 \text { on } \partial \Omega
$$

where $\omega=2 \pi f$ is the pulsation, $v$ is an unknown electric scalar potential, $\sigma$ is the conductivity of tissues [5] and a is a known source magnetic vector potential which can be either computed numerically if a model of the source is available, or determined from available measurements [4], [6], [7]. Equation (1) is most often solved by using the SPFD [8], FIT [9] or FEM method [10], [11]. The main reason which hinders using FEM in dosimetric computations is the difficulty of generating

Manuscript received December 1, 2012; revised August 26, 2015. Corresponding author: R. Scorretti (email:riccardo.scorretti@ec-lyon.fr). a quality non conform mesh of the human body. Unfortunately, such computations are time-consuming, as the human body has to be discretized in detail, and problems with several millions of unknowns have to be solved for each exposure situation. One way to solve this issue is to use GPUs to speed-up the inversion of the linear system, which is the dominant step in dosimetric computations [12].

In this work we present a numerical dosimetry method based on reduced basis, by which real-time computations can be foreseen. The method itself is presented in section II. Application to a simplistic test-case and a more realistic wireless charging system (WCS) for vehicles are presented in section III.

\section{METHOD}

The computational strategy is illustrated in figure 1 . The source is modelled as an equivalent external source multipole [13], the coefficients of which $q_{n}^{(s)}$ can be obtained either by fitting it from experimental or numerical data:

$$
\begin{aligned}
\mathbf{a}^{(\mathbf{s})}(\mathbf{x}) & =\sum_{n=0}^{N} q_{n}^{(s)} \mathbf{a}^{(\mathbf{s})}{ }_{n}(\mathbf{x}) \\
& =\sum_{l=1}^{L} \sum_{m=-l}^{l} q_{l m}^{(s)} \frac{\mu_{0}}{l+1} r^{-(l+1)} \sqrt{l(l+1)} \mathbf{X}_{l m}(\mathbf{x})
\end{aligned}
$$

This external multipole allows to compute $\mathbf{a}^{(\mathbf{s})}$, and hence $\mathbf{b}^{(\mathbf{s})}=\operatorname{curl}^{(\mathbf{s})}$ outside a sphere of radius $R_{s}$. Starting from this multipole, a second multipole of the same order is obtained:

$$
\begin{aligned}
\mathbf{a}^{(\mathbf{v})}(\mathbf{x}) & =\sum_{n=0}^{N} q_{n}^{(v)} \mathbf{a}^{(\mathbf{v})}(\mathbf{x}) \\
& =\sum_{l=1}^{L} \sum_{m=-l}^{l} q_{l m}^{(v)} \frac{\mu_{0}}{l+1} r^{l} \sqrt{l(l+1)} \mathbf{X}_{l m}(\mathbf{x})
\end{aligned}
$$

This second multipole is an internal one, that is it allows to compute $\mathbf{a}^{(\mathbf{v})}$ and $\mathbf{b}^{(\mathbf{v})}=\operatorname{curl}^{(\mathbf{v})}$ inside a sphere or radius $R_{v}$ centered on the "victim" (aka the human body). As such, this method can be used as long as: i) the source and the victim are both entirely contained inside their respective validity 


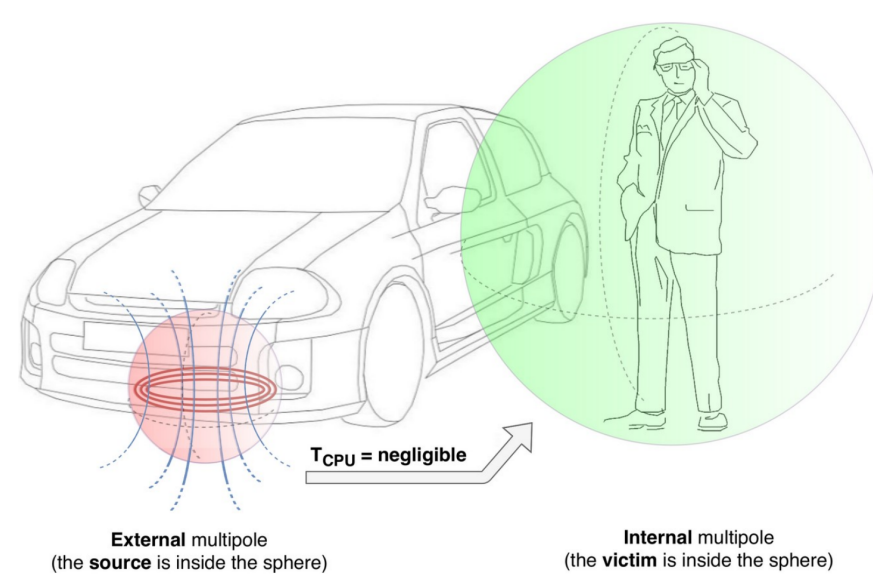

Fig. 1. Sketch of the methodology

spheres, ii) the two spheres don't intersect, iii) the space where the magnetic field is computed by using multipoles doesn't contain any magnetic material or electrical current. By using (3) into (1) one sees that the electric field inside the human body writes:

$$
\mathbf{e}=\sum_{n} q_{n}^{(v)} \mathbf{e}_{n}
$$

where $\mathbf{e}_{n}=-j \omega \mathbf{a}^{(\mathbf{v})}{ }_{n}-\operatorname{grad} v_{n}$ are the elements of a reduced basis, which can be computed off-line by solving:

$$
\operatorname{div}\left[\sigma\left(j \omega \mathbf{a}^{(\mathbf{v})}{ }_{n}+\operatorname{grad} v_{n}\right)\right]=0
$$

The key point is that the coefficients $q_{n}^{(v)}$ of this internal (victim) multipole can be obtained practically at no cost from the coefficients $q_{n}^{(s)}$ of the source multipole. Hence, after having identified the source multipole, it is possible to compute the coefficients $q_{n}^{(v)}$ and then, by using (4), the internal electric field for any position of the human body, provided that conditions i-iii hold. In this work (5) is solved by the FEM method by using the software GetDP [14], [10], but any other method can be used as well.

\section{Applications}

In order to assess the effectiveness and the accuracy of the method, we performed some preliminary tests with a spherical phantom, then we simulated a more realistic situation with an anatomical phantom exposed to the stray field of a WCS. In all computations, the results obtained by (4) have been compared with a reference computation, performed by solving directly (1) with the true magnetic vector potential a.

In our work, the source magnetic field and vector potential have been obtained numerically by classical FEM modelling of the source.

\section{A. Preliminary test}

In order to perform some preliminary tests, we simulated the exposure of a spherical homogeneous phantom (radius = $1 \mathrm{~m}$, conductivity $=1 \mathrm{~S} / \mathrm{m}$ ) to the field generated by a circular coil (radius $=0.1 \mathrm{~m}$, current $I=1 \mathrm{~A}$ ). The center of the coil is at $1.5 \mathrm{~m}$ from the center of the sphere. The axis of the coil

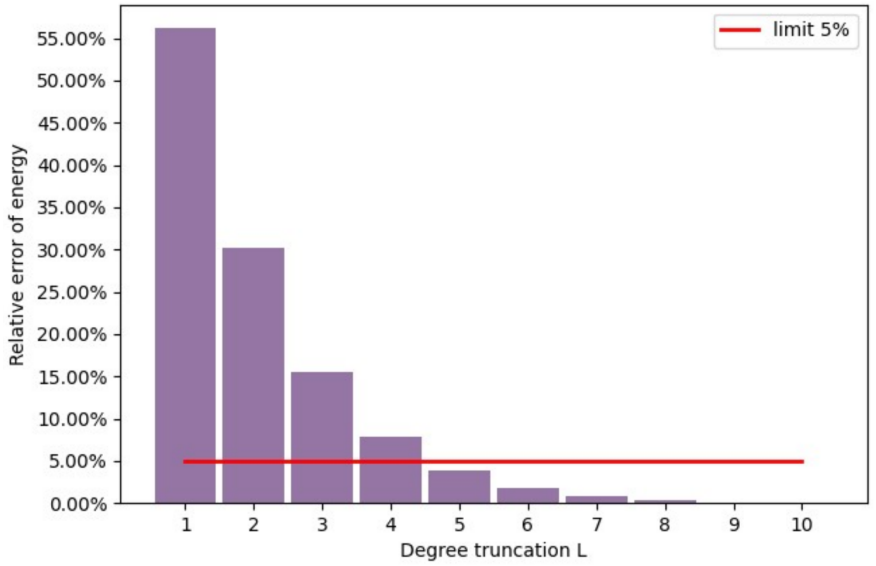

Fig. 2. Relative error as a function of the order of the multipole. The red line represents the $5 \%$ threshold.

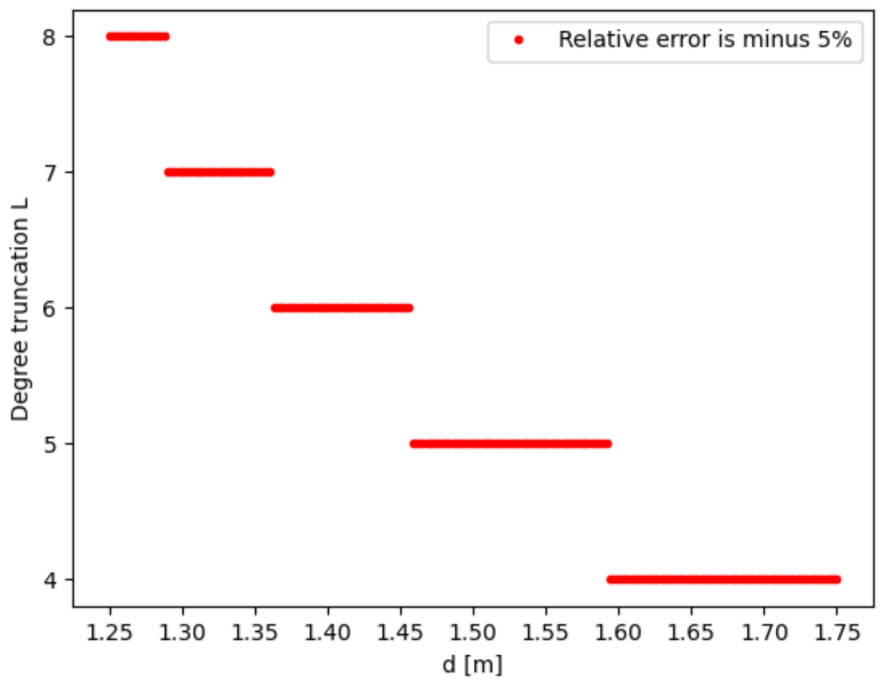

Fig. 3. Minimum order of multipoles required to achieve an accuracy of $5 \%$ as a function of the distance between the centers of the coil and of the sphere.

coincides with a symmetry axis of the sphere. The magnetic field to which the victim is exposed is computed by using (3), and compared with the reference solution. The relative error $\mathcal{E}$ on the magnetic energy with respect of the order $L$ of multipoles is plotted figure 2:

$$
\mathcal{E}=\frac{\left|\sum_{n} \frac{q_{n}^{(v)}}{2 \mu_{0}} \int\left\|\operatorname{curl} \mathbf{a}^{(\mathbf{v})}{ }_{n}\right\|^{2}-\frac{1}{2 \mu_{0}} \int\|\operatorname{curl} \mathbf{a}\|^{2}\right|}{\frac{1}{2 \mu_{0}} \int\|\operatorname{curl} \mathbf{a}\|^{2}}
$$

We observe that in this configuration a minimum order $L=5$ (that is, 15 terms of the reduced basis) is enough to obtain a relative error of less than $5 \%$.

Figure 3 represents the minimum order $L$ of multipoles which is required to achieve an accuracy of at least 5\%. Without surprise, it can be observed that the closer the source, the higher is the minimum order. However, notice that a multipole of the $8^{\text {th }}$ order corresponds to only 36 terms of the reduced basis, which is still acceptable.

The observed speed-up with respect to a standard resolution is of approximately 60 . Notice that the computation performed with GetDP which is a stable and optimized software, whereas 

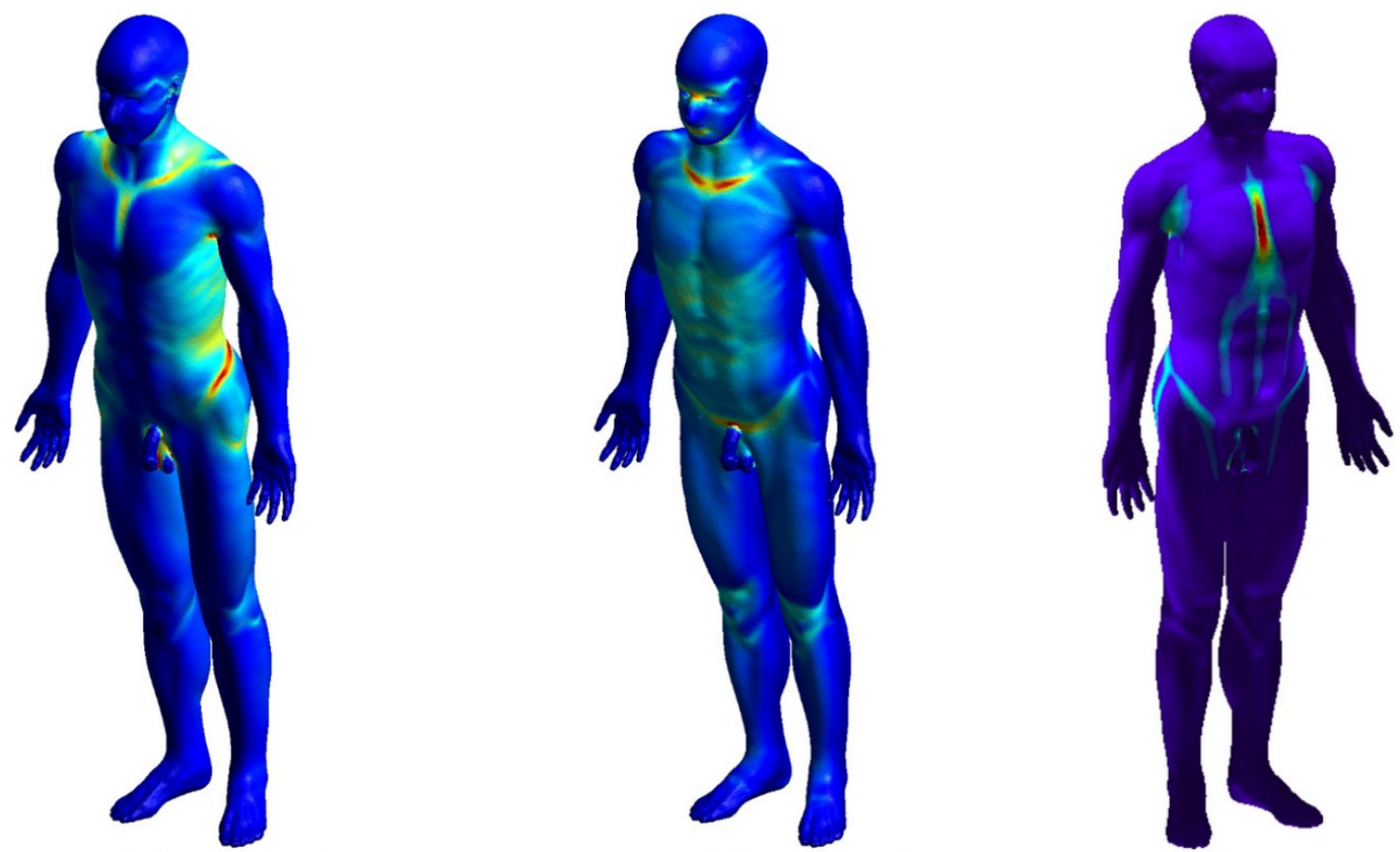

Fig. 4. Low-order terms of the reduced basis of the electric field (from left to right: $\left\|\mathbf{e}_{(1,-1)}\right\|,\left\|\mathbf{e}_{(1,1)}\right\|$ and $\left\|\mathbf{e}_{(1,0)}\right\|$ on the surface of the body).

our code is still being developed; hence a higher speed up can be foreseen.

\section{B. Wireless charging system}

WCS of electrical vehicles is a promising technology. The issue of involuntary exposure to stray fields generated by WCS systems is a hot topic which is being actively investigated [12], [15], [16], [17]. We simulated the exposure of an adult close to a WCS coil, which is representative of existing devices. The WCS is composed of two coils at a distance of $10 \mathrm{~cm}$ each other, located on the floor. The human body is standing aside the coils. Due to the constraints of applicability i-iii), so far it has not been possible to simulate the presence of the car body.

The computational phantom used in computations is Alvar [18]. Starting from the voxel dataset, a non conform tetrahedral mesh has been meshed by using in-house code programmed with Matlab and $\mathrm{C}++$ by using CGAL [19]. The mesh is composed of $1.96 \mathrm{M}$ nodes and 41 distinct tissues, which have been modelled by taking into account their own conductivities [5]. A few terms of the reduced basis are depicted in figure 4. It this work, the reduced basis for the anatomical phantom is limited to the $3^{\text {rd }}$ order, that is 9 terms only.

Each computation by using the reduced basis (4) took approximately $15 \mathrm{~s}$, compared to $3056 \mathrm{~s}$ for a standard computation (the observed speed-up is about 200). The relative error on the internal electric field $\|\mathbf{e}\|$, and the maximum, 99\% percentile and average value of $\|\mathbf{e}\|$ are displayed in figure 5. It is observed that in some organs it can be very high, notably in the cerebellar grey matter $(60 \%)$ and in the white matter $(40 \%)$. However, this is only a numerical problem which is due to the fact that in these organs the electric field is very low. This is confirmed by the fact that in organs where the electric field is high, the relative error is comparatively very low (approximately 5\% for the fat and muscles, which are the two tissues with the higher field).

\section{Conclusion}

We developed a method which allows to compute in nearly real-time the internal electric field induced in the human body by low-frequency electrical appliances. The method has been tested with a simplified geometry of the human body (a sphere) and with a realistic anatomical phantom. We find that the results are reasonably close to a reference solution, computed by FEM. In particular, we observe that the relative error is of the order of $5 \%$ in the organs where the electric field is high, which is acceptable, given that the true solution is unknown due to the large uncertainty on conductivities of organs, especially at low frequency [5]. The order $L$ of the reduced basis required to achieve a given accuracy (for instance $1 \%$ ) depends on the source and of the distance of the victim from it. Hence it is impossible to determine $L$ for any generic source / position of the victim.

The conditions of applicability $\mathrm{i}-\mathrm{iii}$ ) hinder the usage of this computational strategy in cases where the victim is very close to the source of field, which of course is a very interesting case. Several strategies can be foreseen to overcome this problem. One could adopt a different reduced basis, for instance by using Proper Orthogonal Decomposition (POD) [20]. The advantage of such a solution would be to remove the constraints on the relative positions of the source and the victim. By using this approach the computation of the coefficients $q_{n}^{(v)}$ from $q_{n}^{(s)}$ would be less straighforward. However this is not a real problem, because the solution of the linear system still dominates the overall computational effort [12]. 

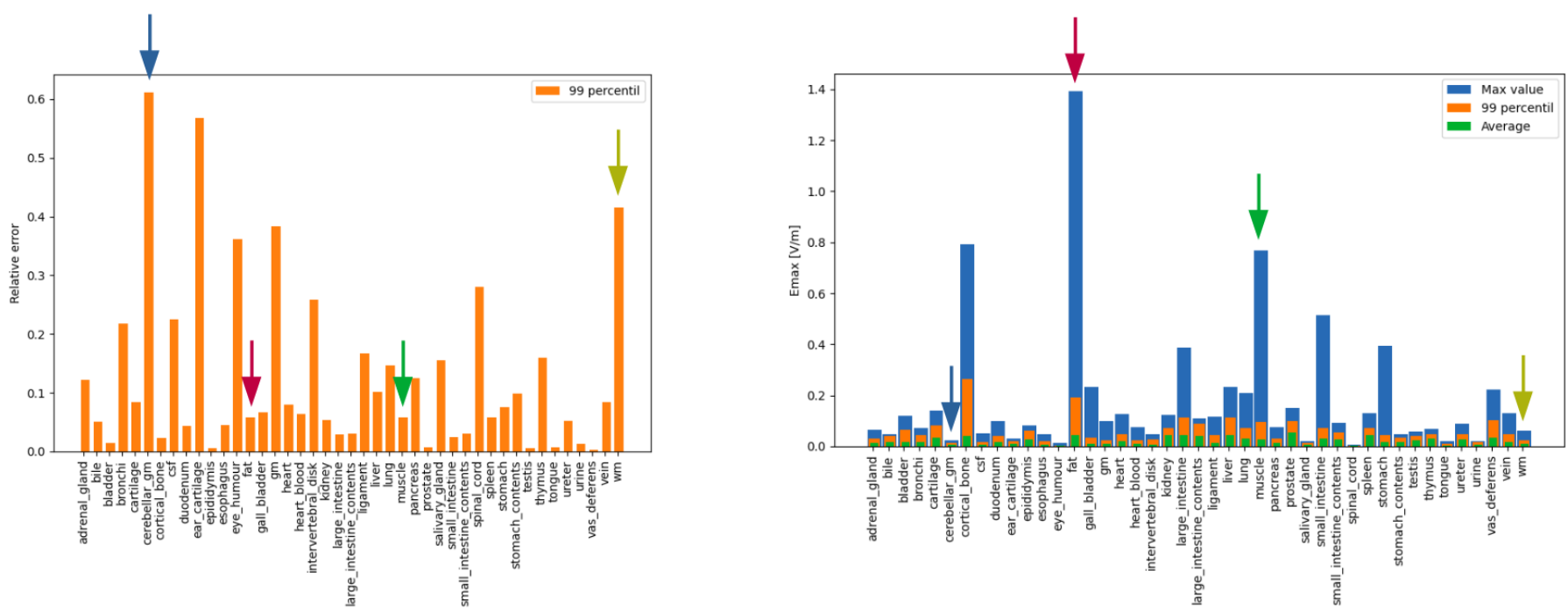

Fig. 5. Relative error on the electric field $\|\mathbf{e}\|$ (left) and max., 99\% percentile and average $\|\mathbf{e}\|$ (right) for each organ. Arrows highlight the following tissues: cerebellar grey matter (blue), fat (red), lungs (green) and white matter (light green).

A different option could be to cover the victim by using a number $K$ of spheres, and to compute the source term (3) on each sphere indepentendly. This approach would allow to compute seamlessly the coefficients $q_{n, k}^{(v)}$ with $k \in[1 ; K]$, but rises the issue of how to deal with the junctions between individual spheres.

Another improvement would be to take into account the anisotropy of muscles, white matter and possibly other tissues. Notice that this is not an issue from the numerical point of view (the problem is still linear): the real problem is to obtain and to deal with the anatomical information about anisotropy.

\section{ACKNOWLEDGMENT}

The authors would like to thank Région Auvergne RhôneAlpes and EMCC (18004892 01 - 4090).

\section{REFERENCES}

[1] ICNIRP, "Guidelines for limiting exposure to time-varying electric and magnetic fields ( $1 \mathrm{~Hz}$ to $100 \mathrm{kHz}$ )," Health physics, vol. 99, no. 6, pp. 818-836, 2010.

[2] A. Ahlbom, U. Bergqvist, J. Bernhardt, J. Cesarini, M. Grandolfo, M. Hietanen, A. Mckinlay, M. Repacholi, D. H. Sliney, J. A. Stolwijk et al., "Guidelines for limiting exposure to time-varying electric, magnetic, and electromagnetic fields (up to $300 \mathrm{GHz}$ )," Health physics, vol. 74, no. 4, pp. 494-521, 1998.

[3] "IEEE Standard for Safety Levels with Respect to Human Exposure to Electric, Magnetic, and Electromagnetic Fields, $0 \mathrm{~Hz}$ to $300 \mathrm{GHz}$," IEEE, Tech. Rep.

[4] R. Scorretti, N. Burais, L. Nicolas, and A. Nicolas, "Modeling of induced current into the human body by low-frequency magnetic field from experimental data," IEEE transactions on magnetics, vol. 41, no. 5, pp. 1992-1995, 2005.

[5] C. Gabriel, A. Peyman, and E. H. Grant, "Electrical conductivity of tissue at frequencies below $1 \mathrm{MHz}$," Physics in medicine and biology, vol. 54 , no. 16 , p. 4863, 2009 .

[6] A. Conchin Gubernati, F. Freschi, L. Giaccone, T. Campi, V. De Santis, and I. Laakso, "Comparison of Numerical Techniques for the Evaluation of Human Exposure From Measurement Data," IEEE Transactions on Magnetics, vol. 55, no. 6, pp. 1-4, Jun. 2019.

[7] Y. L. Diao, W. N. Sun, Y. Q. He, S. W. Leung, and Y. M. Siu, "Equivalent magnetic vector potential model for low-frequency magnetic exposure assessment," Physics in Medicine \& Biology, vol. 62, no. 19, pp. 79057922, Sep. 2017.
[8] K. Aga, A. Hirata, and I. Laakso, "Relationship Between In-Situ Electric Field and External Magnetic Field Strength in Human Models-Rational of IEEE C95.6 Standard Revisited," in 2018 International Symposium on Electromagnetic Compatibility (EMC EUROPE). Amsterdam: IEEE, Aug. 2018, pp. 515-520.

[9] F. Freschi, L. Giaccone, V. Cirimele, and A. Canova, "Numerical assessment of low-frequency dosimetry from sampled magnetic fields," Physics in Medicine \& Biology, vol. 63, no. 1, p. 015029, Dec. 2017.

[10] R. Scorretti, R. V. Sabariego, L. Morel, C. Geuzaine, N. Burais, and L. Nicolas, "Computation of Induced Fields Into the Human Body by Dual Finite Element Formulations," IEEE Transactions on Magnetics, vol. 48, no. 2, pp. 783-786, Feb. 2012.

[11] Z. M. Tech, "Sim4Life," https://zmt.swiss/sim4life.

[12] N. Haussmann, M. Zang, R. Mease, M. Clemens, B. Schmuelling, and M. Bolten, "Towards Real-Time Magnetic Dosimetry Simulations for Inductive Charging Systems," arXiv:2010.12879 [cs], Oct. 2020.

[13] B. Stout, "Spherical harmonic Lattice Sums for Gratings," p. 46.

[14] C. Geuzaine, "GetDP: A general finite-element solver for the de Rham complex," PAMM, vol. 7, no. 1, pp. 1010603-1010 604, Dec. 2007.

[15] I. Liorni, O. Bottauscio, R. Guilizzoni, P. Ankarson, J. Bruna, A. Fallahi, S. Harmon, and M. Zucca, "Assessment of Exposure to Electric Vehicle Inductive Power Transfer Systems: Experimental Measurements and Numerical Dosimetry," Sustainability, vol. 12, no. 11, p. 4573, Jun. 2020.

[16] I. A. Shah and H. Yoo, "Assessing Human Exposure With Medical Implants to Electromagnetic Fields From a Wireless Power Transmission System in an Electric Vehicle," IEEE Transactions on Electromagnetic Compatibility, vol. 62, no. 2, pp. 338-345, Apr. 2020.

[17] V. De Santis, T. Campi, S. Cruciani, I. Laakso, and M. Feliziani, "Assessment of the Induced Electric Fields in a Carbon-Fiber Electrical Vehicle Equipped with a Wireless Power Transfer System," Energies, vol. 11, no. 3, p. 684, Mar. 2018.

[18] I. Laakso and L. Tuukka, "Alvar," https://version.aalto.fi/gitlab/ilaakso/alvar.

[19] C. Jamin, P. Alliez, M. Yvinec, and J.-D. Boissonnat, "CGALmesh: A Generic Framework for Delaunay Mesh Generation," ACM Transactions on Mathematical Software, vol. 41, no. 4, pp. 1-24, Oct. 2015.

[20] R. Pinnau, "Model reduction via proper orthogonal decomposition," in Model Order Reduction: Theory, Research Aspects and Applications. Springer, 2008, pp. 95-109. 